\title{
A study on the dielectric properties of palm oil and coconut oil
}

\begin{abstract}
One of the important parameter for insulating fluid is the dielectric properties. With the introduction of vegetable oil as possible alternative fluid for transformers application, it is essential to ensure this type of oil could meet the dielectric properties requirement. In this paper, a study is carried out to investigate the dielectric properties of Palm Oil (PO) and Coconut Oil (CO). The type of PO used in this study is Refined, Bleached and Deodorized Palm Oil (RBDPO) Olein. Several parameters such as AC breakdown voltage, dielectric dissipation factor, relative permittivity and resistivity are examined for both as-received and dried samples. It was found that there are differences on the dielectric dissipation factor and resistivity between RBDPO and $\mathrm{CO}$ and the dielectric properties performances of all samples are improved after subjected to the drying procedure.
\end{abstract}

Keyword: AC breakdown voltage; Coconut oil; Dielectric dissipation factor; Dielectric insulating fluid; Palm oil; Relative permittivity; Resistivity; Transformers 